\title{
Six Degrees-of-freedom Dilution of Precision for Integrity of Camera-based Localization
}

\author{
Chen Zhu, Christian Steinmetz, Boubeker Belabbas, and Michael Meurer \\ Institute of Communications and Navigation, \\ German Aerospace Center (DLR), Oberpfaffenhofen, Germany \\ Email: \{Chen.Zhu, Christian.Steinmetz,Boubeker.Belabbas, Michael.Meurer\}@dlr.de
}

\section{BIOGRAPHY}

Chen Zhu is a full-time research fellow at the Institute of Communications and Navigation, German Aerospace Center (DLR) since April 2018, after submitting his Ph.D. dissertation at Technical University of Munich, in Munich, Germany. He received his B.Sc. in Automation Engineering from Tsinghua University, in Beijing, China in 2009, and his M.Sc. in Communications Engineering in 2011 from Technical University of Munich. His research interests include visual navigation, multi-sensor fusion, robotic swarm navigation, and he is focusing on the integrity of the techniques.

Christian Steinmetz obtained a Bachelor of Science degree in Electrical Engineering and Information Technology at the Technical University of Munich. He is currently studying the consecutive Master's program there. For his Master's thesis, he joined the Department of Navigation at the Institute of Communications and Navigation, German Aerospace Center (DLR).

Boubeker Belabbas obtained a MSc. Degree in Mechanical Engineering from ENSEM in Nancy (France) and a specialized MSc. Degree in Aerospace Mechanics from SUPAERO in Toulouse (France). He joined DLR in 2001 and developed the navigation integrity activities. He now leads the GNSS integrity team within the Department of Navigation at the Institute of Communications and Navigation. His field of competence includes GNSS with augmentations (SBAS, GBAS), Advanced Receiver Autonomous Integrity Monitoring, GNSS/INS Hybridization with integrity, and Alternative Positioning Navigation and Timing.

Prof. Michael Meurer is the director of the Department of Navigation at the German Aerospace Center (DLR) and of the Center of Excellence for Satellite Navigation. In addition, he is a full professor of electrical engineering and director of the Chair of Navigation at the RWTH Aachen University. His current research interests include GNSS signals, GNSS receivers and navigation for safety-critical applications.

\footnotetext{
ABSTRACT

Camera-based visual navigation techniques are capable of providing high precision localization solution, and play an important role in challenging urban environments, e.g., indoor or urban canyons, where satellite navigation has significantly degraded performance. For safety critical applications, the integrity monitoring of visual navigation methods is necessary. In order to calculate the localization error for integrity measures such as protection level, it is essential to investigate the geometric impacts. The dilution of precision concept from satellite navigation can be analogized to camera sensors. However, according to the measurement model of cameras, the attitude of the camera has influences on the uncertainty of the position estimates, and vice versa. This work investigates the geometric impact on camera-based localization by using a six degrees-of-freedom dilution of precision including both position and attitude. The attitude is parameterized using Rodrigues parameters instead of Euler angles to avoid Gimbal lock. The geometric impact is analyzed in different scenarios using real images.
} 


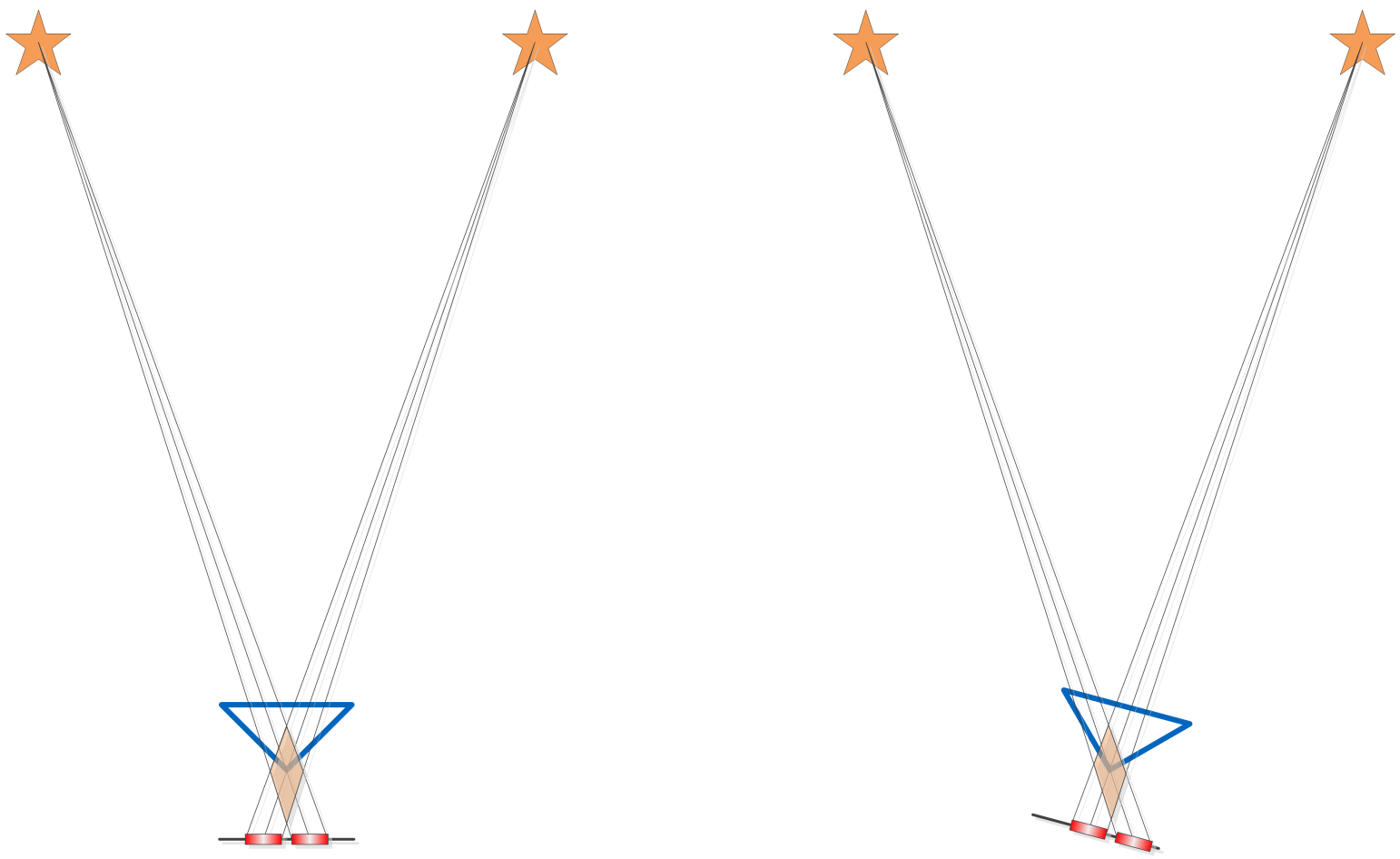

Figure 1: Impact of camera attitude on positioning uncertainty

\section{INTRODUCTION}

In several scenarios such as indoor environments or urban canyons, the signals of global navigation satellite systems (GNSS) have significantly degraded quality or are even unavailable. However, given the abundant visual cues and landmarks in urban scenarios, camera-based visual navigation techniques are capable of providing six degrees-of-freedom (DoF) pose estimates. Including cameras as additional navigation sensor may improve the navigation system performance in accuracy, availability, continuity and integrity. However, quantitative integrity monitoring of camera-based localization is not yet a well-solved problem. In order to analyze the localization error of visual navigation methods for integrity monitoring, it is an essential step to calculate the geometric impacts on the pose estimates. Moreover, the error source can be better investigated by decoupling the geometric impact and noise impact on the localization error. The dilution of precision (DOP) concept from satellite navigation [1] can be analogized to digital camera sensors. However, according to the measurement model of cameras, the attitude of the camera has influences on the uncertainty of the position estimates, and vice versa. Fig. 1 illustrates the impact with a simple two dimensional example. It can be seen that in the two cases, the camera and landmark location as well as the measurement covariance are the same, but the difference in the camera attitude results in distinct uncertainty domains in the camera location estimation. Consequently, it is insufficient to calculate the position DOP (PDOP) only, but also the attitude DOP (AtDOP). The attitude DOP concept is proposed for GNSS and radar fusion by Johnson et al. in [2]. However, due to the correlation of the position and the attitude in the camera measurement model, both PDOP and AtDOP should be calculated jointly in a 6 dimensional space.

This work proposes a DOP definition for visual positioning. The position and attitude are jointly considered in 6 DoF poses. The attitude of the camera is parameterized using Rodrigues parameters, which avoids Gimbal locks and provides a concise metric for calculating AtDOP easily.

\section{MEASUREMENT MODEL}

In order to investigate the geometric impact on camera-based localization, state-of-the-art pinhole model is used as the measurement model of the camera.

Fig. 2 illustrates the system model of a pinhole camera. The projection of a point with coordinates $\vec{X}_{i}^{(W)} \in \mathbb{R}^{3}$ to the camera 


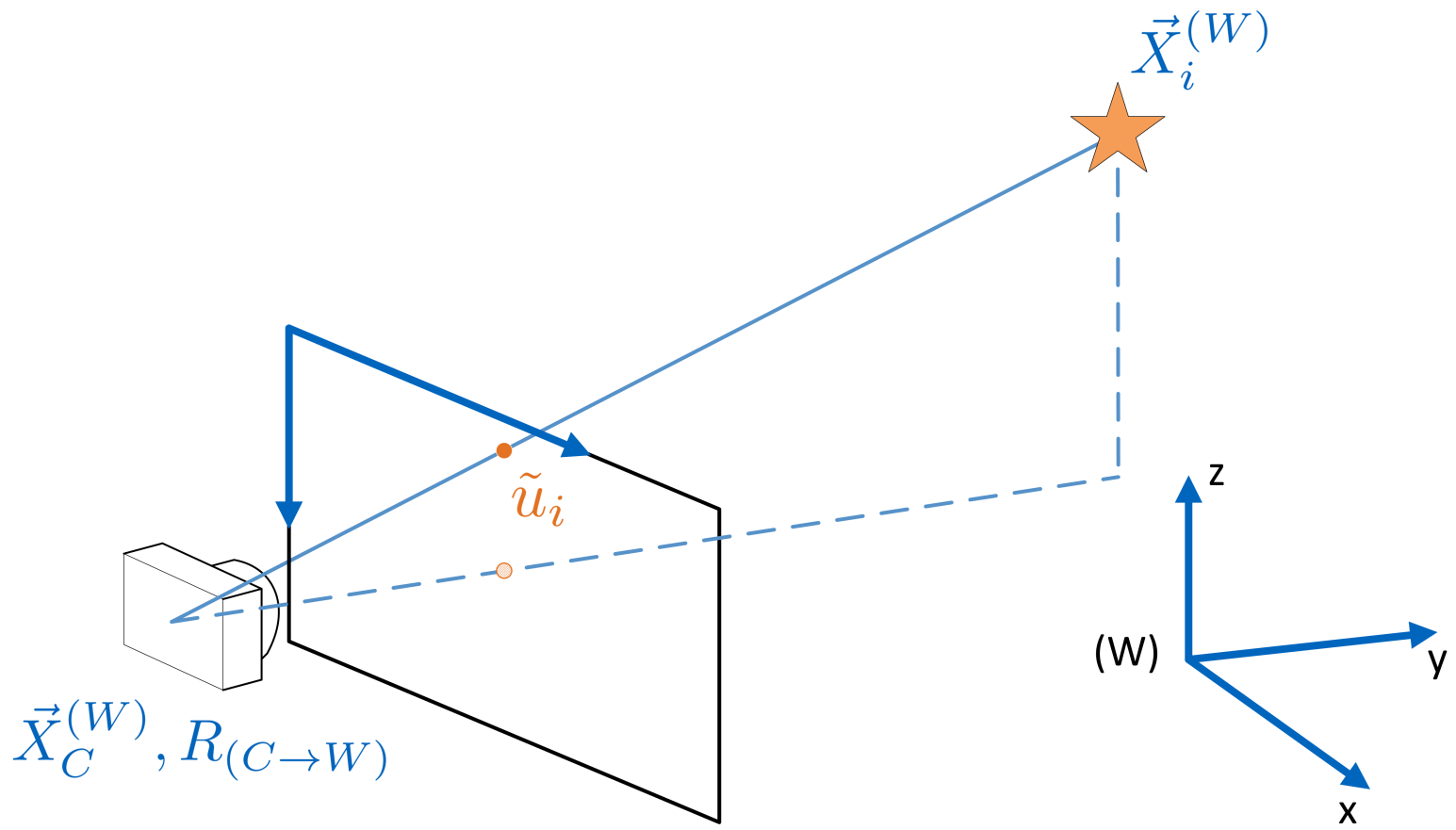

Figure 2: System model of a pinhole camera

is described as

$$
\tilde{u}_{i}=K R_{(C \rightarrow W)}^{T}\left(\vec{X}_{i}^{(W)}-\vec{X}_{C}^{(W)}\right),
$$

where $\vec{X}_{C}^{(W)}$ is camera position in the global reference frame $(W)$, and $R_{(C \rightarrow W)} \in \mathbf{S O}(3)$ is the rotation matrix between the camera body frame $(C)$ and frame $(W)$. The matrix $K$ is called camera intrinsic matrix, the parameters in which are only dependent on the camera and the lens used. $\tilde{u}_{i}=d\left[u_{x}, u_{y}, 1\right]^{T}$ is the location of the point $i$ on the image plane in homogeneous coordinates, where $d$ is the depth of the point, i.e., the distance of the point to the image plane. The depth can be calculated by

$$
d=[0,0,1] R_{(C \rightarrow W)}^{T}\left(\vec{X}_{i}^{(W)}-\vec{X}_{C}^{(W)}\right) .
$$

In the remainder of this paper, a superscript with parentheses ${ }^{(\cdot)}$ is used to denote the reference frame in which the vector is represented. Vectors such as $\vec{X}_{i}^{(W)} \in \mathbb{R}^{3}$ with geometric meanings are written with an arrow. The homogeneous coordinates in the extended Euclidean plane are written as $\tilde{r} \in \mathbb{P}^{2}$.

For a corner extracted from the measurement image, the noisy 2D location of the corner can be modelled as

$$
\mu_{i}=u_{i}+n_{i}=\left[u_{x}, u_{y}\right]^{T}+n_{i}=\frac{1}{d}\left[\begin{array}{lll}
1 & 0 & 0 \\
0 & 1 & 0
\end{array}\right] \tilde{u}_{i}+n_{i},
$$

where $n_{i}$ is zero-mean Gaussian noise with covariance matrix $\Sigma_{n i}$.

\section{PARAMETERIZATION OF THE CAMERA ATTITUDE}

We parameterize the camera attitude using Rodrigues parameters $r=\left[r_{1}, r_{2}, r_{3}\right]^{T}=\theta e$, which can be related to the rotation matrix by Rodrigues rotation formula as

$$
R_{(C \rightarrow W)}=I_{3} \cos (\theta)+\lfloor e\rfloor_{\times} \sin (\theta)+(1-\cos (\theta)) e e^{T}=I_{3}+\lfloor e\rfloor_{\times} \sin (\theta)+(1-\cos (\theta))\lfloor e\rfloor_{\times}^{2},
$$

where $I_{3}$ is a 3 -dimensional identity matrix, and $\lfloor e\rfloor_{\times}$is the skew symmetric matrix using the normalized unit vector $e=r /\|r\| \in \mathbb{R}^{3}$ as none-zero entries. The operator $[\cdot]_{x}$ is defined as

$$
\lfloor e\rfloor_{\times}=\left[\begin{array}{ccc}
0 & -e_{3} & e_{2} \\
e_{3} & 0 & -e_{1} \\
-e_{2} & e_{1} & 0
\end{array}\right] .
$$


It should be mentioned that the three Roderigues parameters are actually the coefficients of the Lie algebra corresponding to the element of $\mathbf{S O}(3)$ group. The rotation can equivalently represented as

$$
R_{(C \rightarrow W)}=\exp \left(\lfloor r\rfloor_{\times}\right)
$$

where $\exp (\cdot)$ is matrix exponential operator. Such minimal parameterization of attitude is convenient for operations on manifold. It is mathematically elegant, and is capable of avoiding Gimbal lock problem of Euler angle parameterization. In addition, the norm of the attitude vector

$$
\|r\|=\sqrt{r_{1}^{2}+r_{2}^{2}+r_{3}^{2}}=\theta
$$

is naturally a metric of the rotation (proportional to the geodesic on the $\mathbf{S O}(3)$ group) [3].

Consequently, we can parameterize the camera pose with a $6 \mathrm{DoF}$ vector

$$
\xi=\left[\begin{array}{c}
\vec{X}_{C}^{(W)} \\
r
\end{array}\right] \in \mathbb{R}^{6}
$$

\section{DILUTION OF PRECISION FOR VISUAL POSITIONING}

Given the 3D point coordinates $\vec{X}_{i}^{(W)}$, the feature location measurements can be related to the camera pose by

$$
\mu_{i}=\pi_{i}(\xi)+n_{i}
$$

with

$$
\pi_{i}(\xi)=\frac{\left[\begin{array}{lll}
1 & 0 & 0 \\
0 & 1 & 0
\end{array}\right] K R_{(C \rightarrow W)}^{T}\left(\vec{X}_{i}^{(W)}-\vec{X}_{C}^{(W)}\right)}{[0,0,1] R_{(C \rightarrow W)}^{T}\left(\vec{X}_{i}^{(W)}-\vec{X}_{C}^{(W)}\right)} .
$$

By stacking the measurements from $N$ feature points into a vector, the measurement equation for visual positioning problem becomes

$$
\mu=\pi(\xi)+n
$$

where

$$
\mu=\left[\begin{array}{c}
\mu_{1} \\
\vdots \\
\mu_{N}
\end{array}\right], \pi(\xi)=\left[\begin{array}{c}
\pi_{1}(\xi) \\
\vdots \\
\pi_{N}(\xi)
\end{array}\right], n=\left[\begin{array}{c}
n_{1} \\
\vdots \\
n_{N}
\end{array}\right]
$$

The pose of the camera can be estimated by minimizing the projected feature location error as

$$
\hat{\xi}=\arg \min _{\xi}\|\mu-\pi(\xi)\|_{\Sigma_{n}}^{2},
$$

where $\Sigma_{n}$ is the covariance matrix of noise vector $n .\|\cdot\|_{\Sigma_{n}}$ denotes the Mahalanobis distance with covariance $\Sigma_{n}$. By linearizing at $\hat{\xi}$, the measurement equation is approximated by

$$
\mu \approx \pi(\hat{\xi})+J_{\pi}(\xi-\hat{\xi})+n,
$$

where $J_{\pi}$ is the Jacobian matrix at linearized point. The nonlinear optimization can be solved iteratively using Gauss-Newton or Levenberg-Marquardt algorithm [4].

The covariance of the estimated parameters can be approximated as

$$
\Sigma_{\xi}=E\left\{(\xi-\hat{\xi})(\xi-\hat{\xi})^{T}\right\}=\left(J_{\pi}^{T} \Sigma_{n}^{-1} J_{\pi}\right)^{-1} .
$$




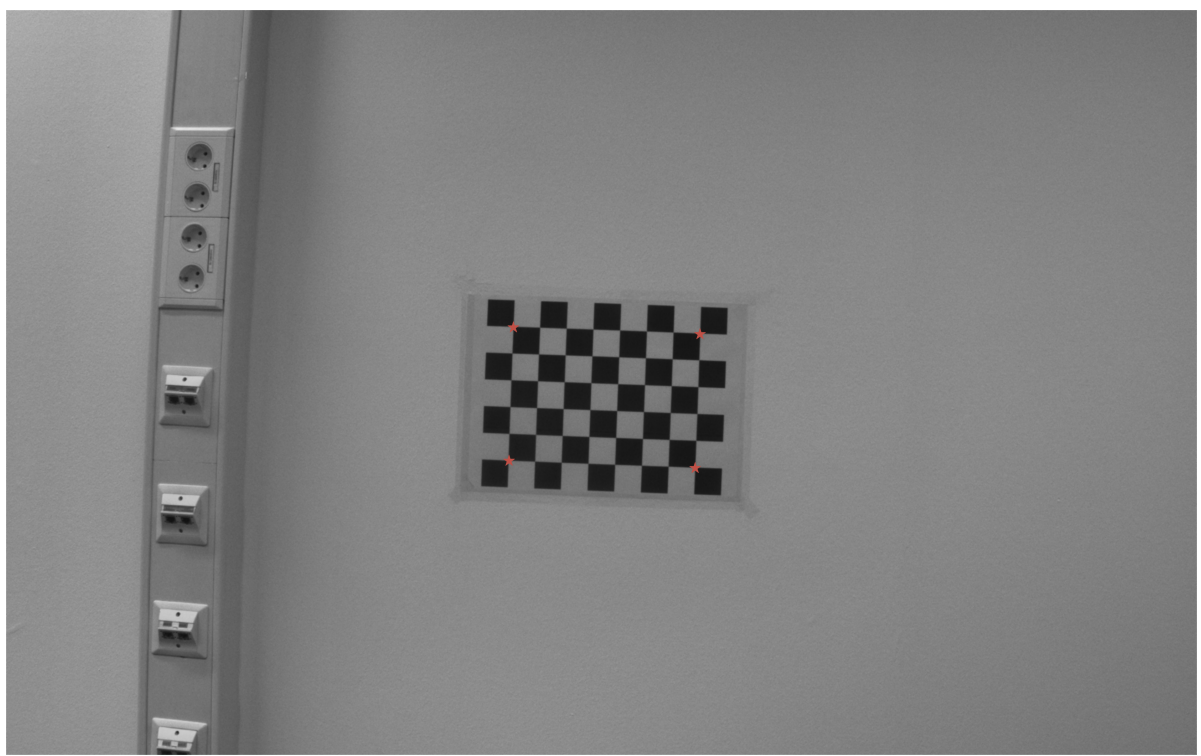

Figure 3: Sample image with marked corners

If we decouple the feature error impact and the geometric impact, the geometric part can be described by matrix $A$ with diagonal elements as the squared 6 DoF dilution of precision of the estimated pose, i.e.,

$$
A=\left(J_{\pi}^{T} J_{\pi}\right)^{-1}=\left[\begin{array}{cccccc}
x D O P^{2} & * & * & * & * & * \\
* & y D O P^{2} & * & * & * & * \\
* & * & z D O P^{2} & * & * & * \\
* & * & * & r_{1} D O P^{2} & * & * \\
* & * & * & * & r_{2} D O P^{2} & * \\
* & * & * & * & * & r_{3} D O P^{2}
\end{array}\right]
$$

where the non-diagonal elements are marked as $*$ for simplicity.

As a result, given a particular geometry between the camera and the landmarks, the 6DoF DOP can be calculated. The first three components corresponds to the position error, and the other three components corresponds to the attitude error. The PDOP and AtDOP for camera-based localization can be defined respectively as

$$
P D O P=\sqrt{x D O P^{2}+y D O P^{2}+z D O P^{2}},
$$

and

$$
A t D O P=\sqrt{r_{1} D O P^{2}+r_{2} D O P^{2}+r_{3} D O P^{2}} .
$$

The squared summation of the attitude components $r_{i} D O P(i=1,2,3)$ is mathematically a metric for the change of the rotation.

\section{RESULTS}

According to the calculation method using Eqn. (16), 6DoF DOP can be calculated for scenarios with different geometries. In order to verify the correctness of the DOP calculation method, and to investigate the geometric impacts on the camera-based positioning, we set up a checkerboard on the wall and change the relative pose between the camera and the checkerboard. In the tests, only the four vertices of the chessboard inner corners are used for visual positioning, as marked in Fig. 3 with red stars. The reference frame is chosen as in Fig. 4.

We first keep the camera attitude fixed and change the distance between the camera and the wall, as demonstrated in Fig. 5.

The calculated PDOP at different distances is shown in Fig. 6 and the calculated AtDOP is shown in Fig. 7. 


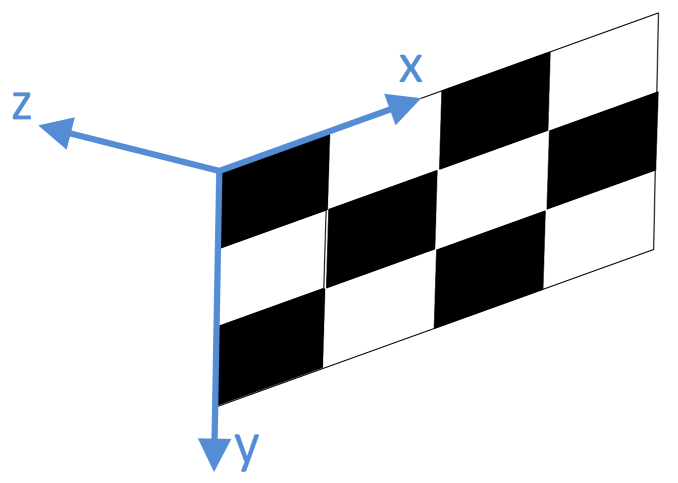

Figure 4: Reference frame in the experiments
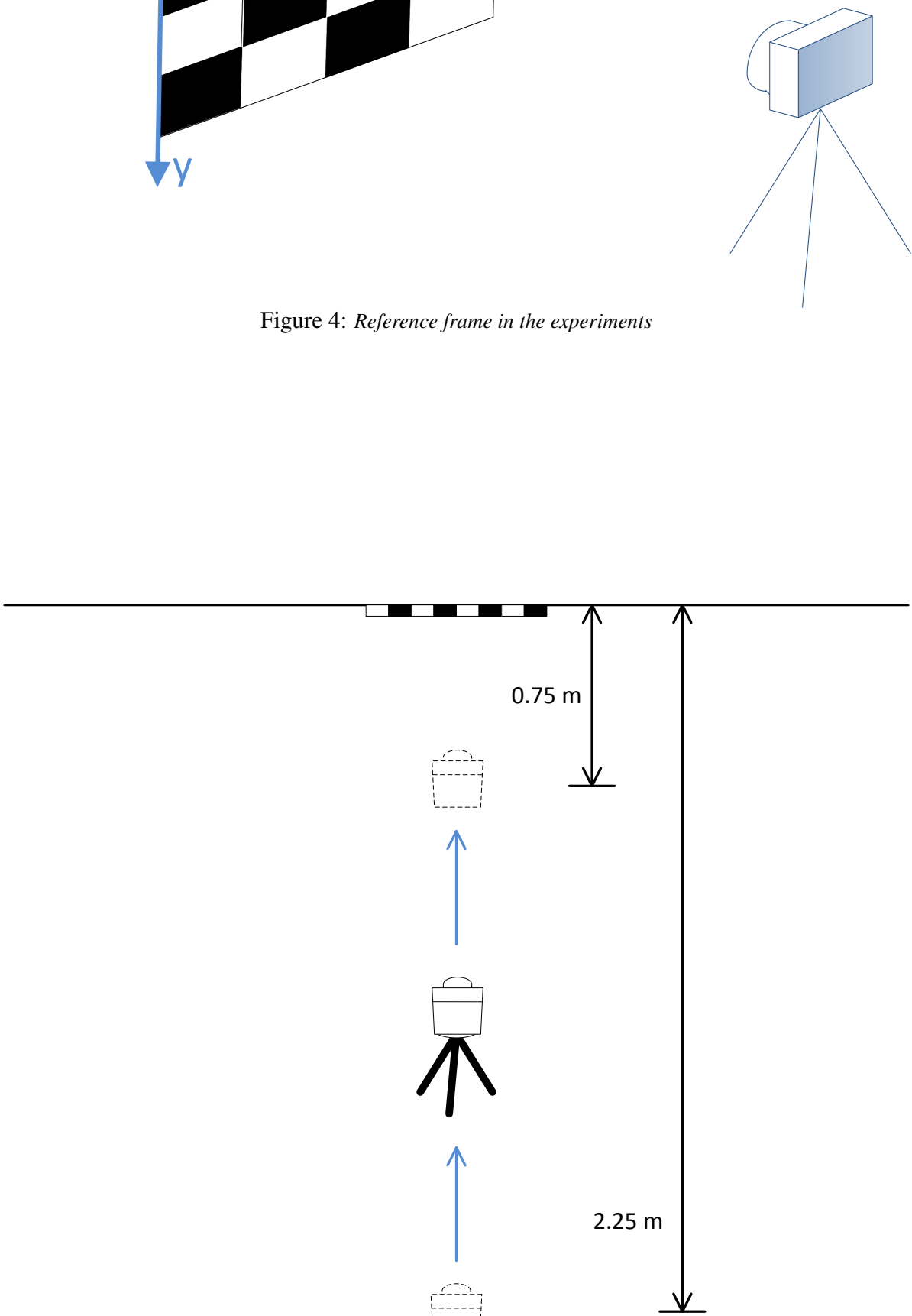

Figure 5: Test scenario 1 


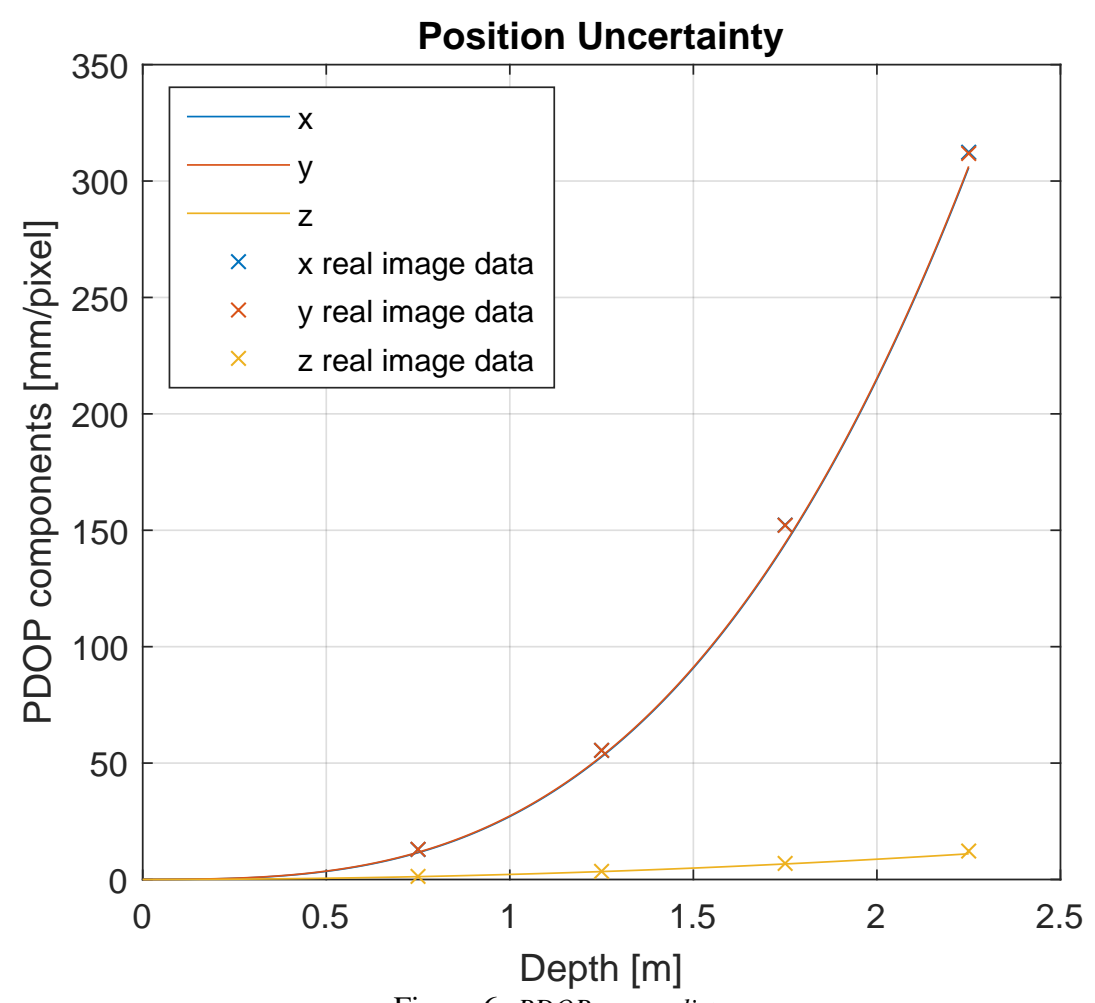

Figure 6: $P D O P$ versus distance

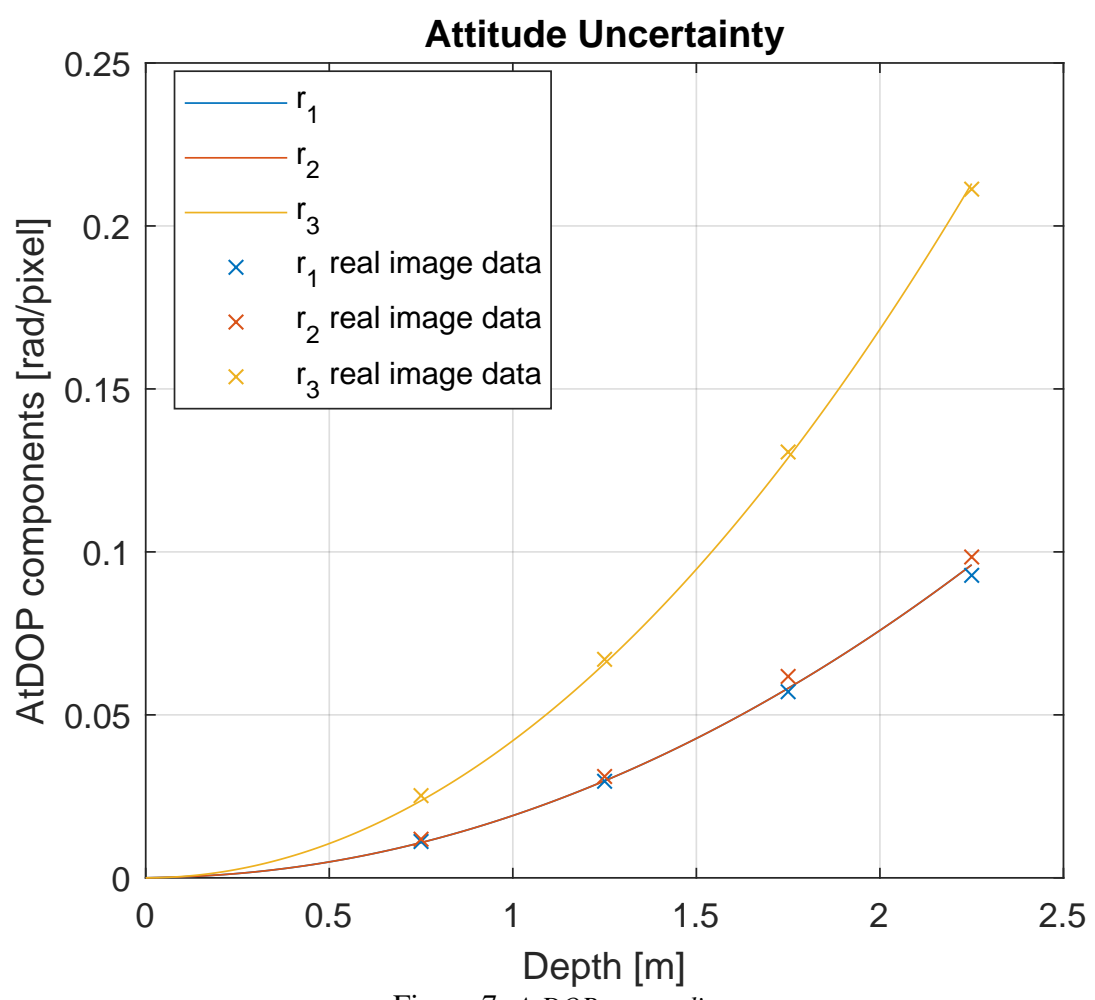

Figure 7: AtDOP versus distance 


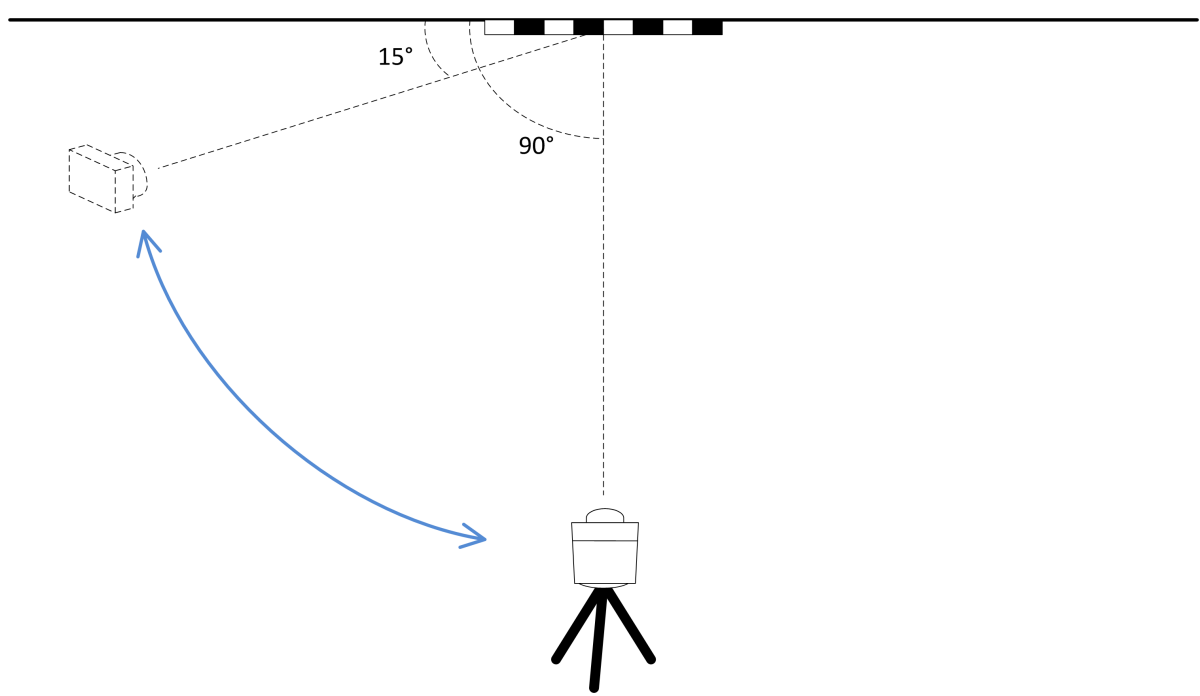

Figure 8: Test scenario 2

The curves with solid line are the theoretical 6DoF DOP for different camera geometries calculated using the proposed method. The samples marked by cross signs are the DOP calculated using real image sets. 2000 measurement images are taken for each camera location. The uncertainty of the estimated camera pose and of the 2D feature locations are extracted from the images statistically. The real image DOP is calculated as the ratio of the pose error standard deviation to the feature error standard deviation. It can be seen from the plots that the samples obtained using real images align well with the curves. Therefore, the proposed 6 DoF DOP calculation method correctly reflects the geometric impact in visual positioning.

We further verify the method by changing the camera view point while keeping the distance between the camera and the checkerboard constant (1.25 meter in this test), as demonstrated in Fig. 8.

The calculated PDOP at different angles of view is shown in Fig. 9 and the calculated AtDOP is shown in Fig. 10.

It can be concluded that the proposed DOP calculation aligns well with the dilution of precision calculated using real measurement images in both scenarios. The PDOP and AtDOP can be applied as a metric of geometric impacts between the camera and the landmarks in visual positioning. It also helps researchers better understand and identify the error sources in visual navigation. 


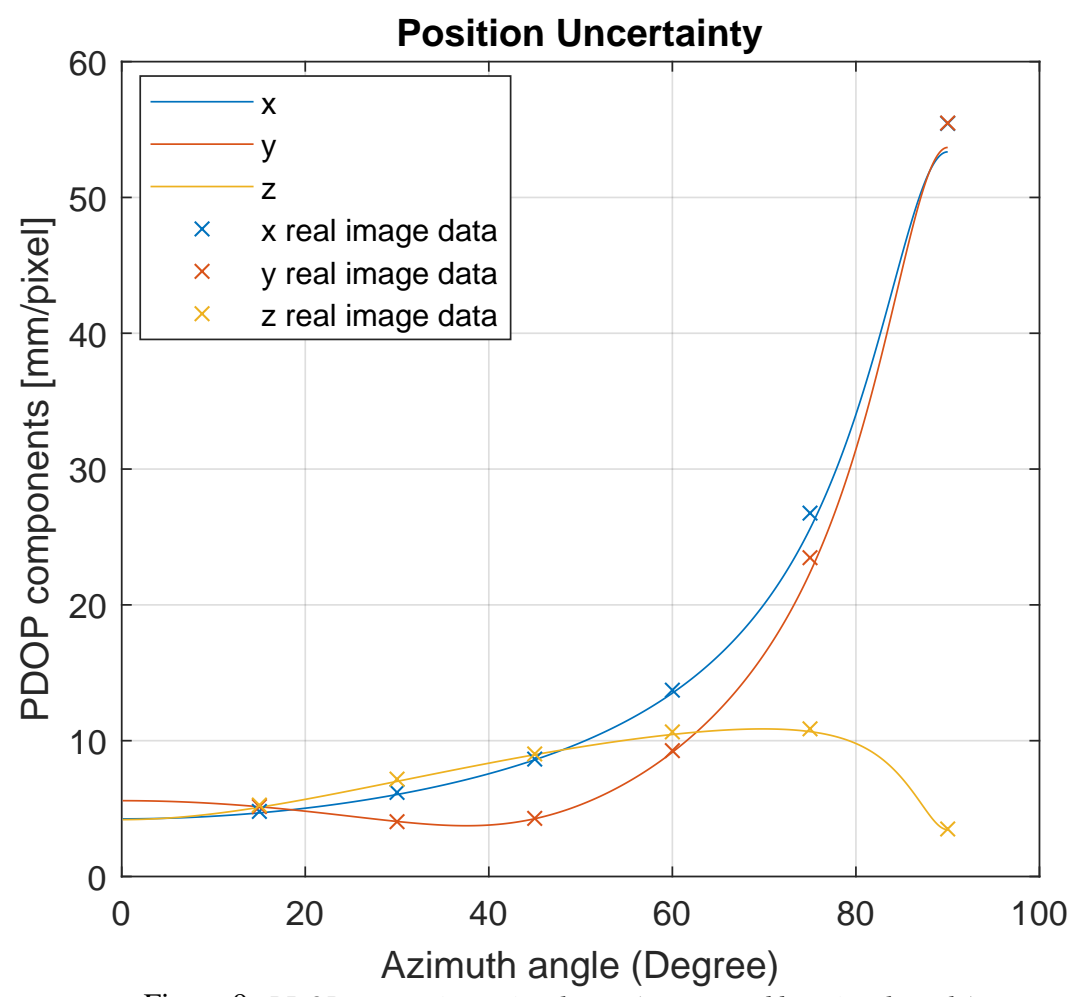

Figure 9: PDOP versus view point change (represented by azimuth angle)

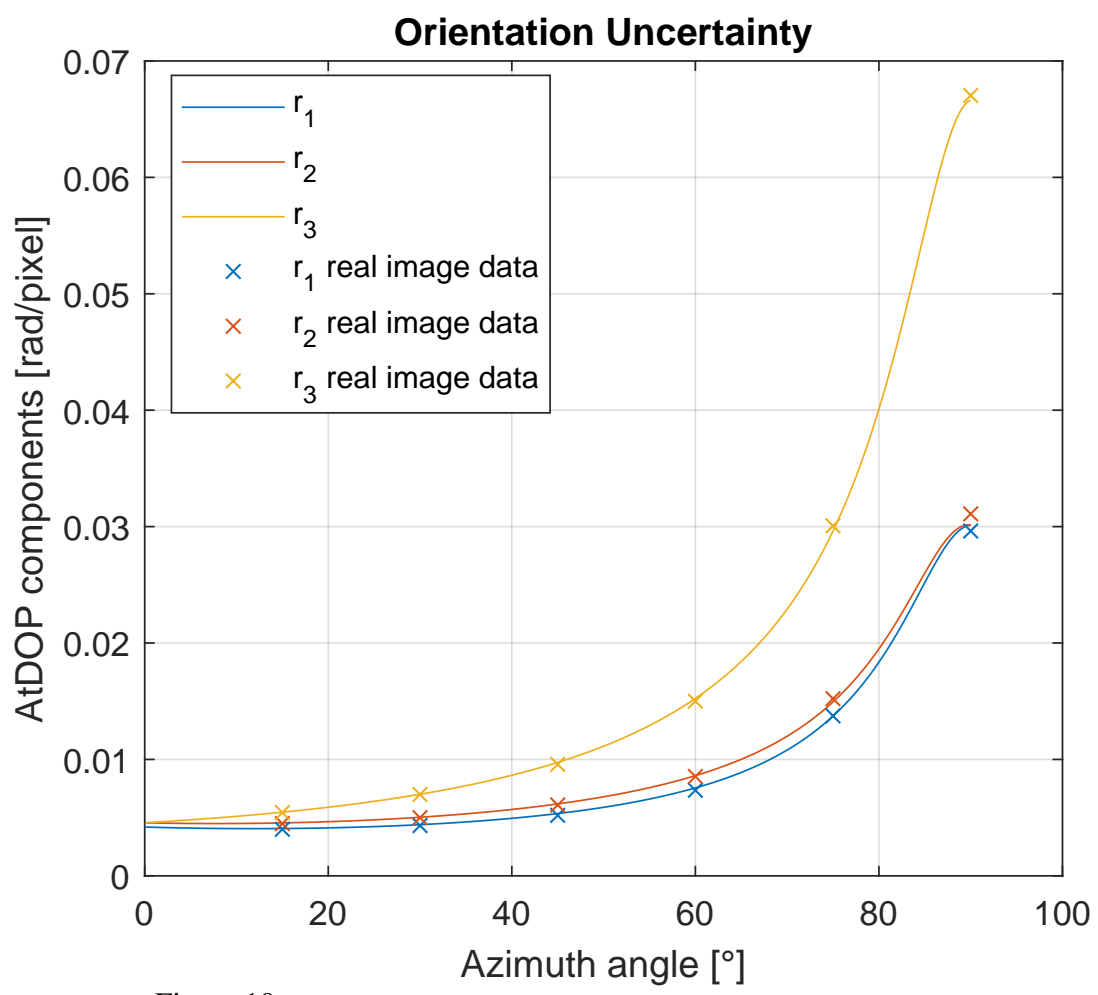

Figure 10: AtDOP versus view point change (represented by azimuth angle) 


\section{REFERENCES}

[1] R. B. Langley et al., "Dilution of precision," GPS world, vol. 10, no. 5, pp. 52-59, 1999.

[2] C. Johnson, E. Ohlmeyer, and T. Pepitone, "Attitude dilution of precision-a new metric for observability of inflight alignment errors," in 18th Applied Aerodynamics Conference, 2000, p. 4277.

[3] D. Q. Huynh, "Metrics for 3d rotations: Comparison and analysis," Journal of Mathematical Imaging and Vision, vol. 35, no. 2, pp. 155-164, Oct 2009. [Online]. Available: https://doi.org/10.1007/s10851-009-0161-2

[4] J. J. Moré, "The levenberg-marquardt algorithm: implementation and theory," in Numerical analysis. Springer, 1978, pp. 105-116. 